\title{
Nonadiabatic losses from radio-frequency-dressed cold-atom traps: Beyond the Landau-Zener model
}

\author{
Kathryn A. Burrows, ${ }^{1}$ Hélène Perrin, ${ }^{2}$ and Barry M. Garraway ${ }^{1}$ \\ ${ }^{1}$ Department of Physics and Astronomy, University of Sussex, Brighton BN1 9QH, United Kingdom \\ ${ }^{2}$ Laboratoire de physique des lasers, CNRS, Université Paris 13, Sorbonne Paris Cité, 99 avenue J.-B. Clément, \\ F-93430 Villetaneuse, France
}

(Received 1 May 2017; published 30 August 2017)

\begin{abstract}
Nonadiabatic decay rates for a radio-frequency-dressed magnetic trap are calculated using Fermi's golden rule: that is, we examine the probability for a single atom to make transitions out of the dressed trap and into a continuum in the adiabatic limit, where perturbation theory can be applied. This approach can be compared to the semiclassical Landau-Zener theory of a resonant dressed atom trap, and it is found that, when carefully implemented, the Landau-Zener theory overestimates the rate of nonadiabatic spin-flip transitions in the adiabatic limit. This indicates that care is needed when determining requirements on trap Rabi frequency and magnetic-field gradient in practical atom traps.
\end{abstract}

DOI: 10.1103/PhysRevA.96.023429

\section{INTRODUCTION}

The control of ultracold atomic systems holds great promise for applications in quantum technology such as sensors for gravity, magnetism, and motion. Magnetic trapping is one of the ways of both trapping and controlling the atoms. However, by introducing radio-frequency (rf) fields $[1,2]$ we can form adiabatic, or dressed, potentials, which offer a high degree of control over trapping topology and hold promise for becoming a standard tool for manipulating atoms and atom interferometry. This particular type of cold-atom trap was suggested by Zobay and Garraway in 2001 [3] and first experimentally achieved in Paris in 2003 [4,5]. The trapping potential is created by the atomic interaction with applied magnetic and rf fields such that the potential depends on the atomic Zeeman state.

However, atoms may be completely lost from the trap if they undergo a transition from a spin state associated with a trapping adiabatic potential to an untrapped spin state. In general, this can happen because an atom travels "too fast" so that the normal adiabatic following cannot take place. A moving atom could experience a rapid change in the local magnetic field amplitude or direction as its position changes. Or the atom could similarly experience a rapid change in the $\mathrm{rf}$ amplitude or polarization as it moves. This is motivated by experiments $[5,6]$ and the need for a greater understanding of nonadiabatic losses as the technique of adiabatic potentials is used in ways that require working to the limits of adiabaticity. In particular, we will present full quantum calculations, but because of the complexity of the problem we make a onedimensional model. Our full problem has gravity present, and so the 1D model is presented for two different orientations which relate to experiments (i.e., horizontal $[7,8]$ and vertical orientations [5]). We then benchmark the 1D model to the much simpler and widely known Landau-Zener model $[9,10]$, which presents a semiclassical approach.

In the related case of static magnetic-field traps, loss rates have been calculated previously $[11,12]$. However, in the present paper a theory is presented for the rate of nonadiabatic transitions between dressed spin states for rf-dressed coldatom traps. In the following Sec. II we will recall the principle of rf-dressed magnetic traps and then we give the predictions of Landau-Zener theory in Sec. III. Section IV presents the quantum treatment of nonadiabatic losses from the trap, which is compared to the predictions of Landau-Zener theory in Sec. V. Finally, we conclude the paper in Sec. VI.

\section{BASICS OF ADIABATIC POTENTIALS}

A single nonrelativistic atom with mass $M$ is trapped in the $z$ direction by an adiabatic potential arising from its interaction with two fields: a static magnetic field and an rf field. The total Hamiltonian describing this problem is given by $[1,2,13]$

$$
\hat{H}_{\mathrm{tot}}=\frac{\hat{p}_{z}^{2}}{2 M}+g_{F} \frac{\mu_{B}}{\hbar} \mathbf{F} \cdot \mathbf{B}(\hat{z})+g_{F} \frac{\mu_{B}}{\hbar} \mathbf{F} \cdot \mathbf{B}_{\mathrm{rf}}(t)+M g \hat{z} .
$$

This forms the 1D model which we study. The first term in the Hamiltonian (1) is the atom's kinetic energy, where $\hat{p}_{z}$ is the momentum operator in the $z$ direction. The second term describes how the atom responds to a static magnetic field $\mathbf{B}(z)$. The total angular momentum of the atom is $\mathbf{F}$ in multiples of $\hbar, \mu_{B}$ is the Bohr magneton, and $g_{F}$ is the Landé factor. The third term in Eq. (1) represents the corresponding interaction with a uniform rf field, and the last term is the gravitational potential of the atom where $g$ is the gravitational acceleration. This last term may be present, or not, depending on the orientation of the direction of 1D trapping with respect to local gravity. We will consider two particular cases: in the case where the trapping direction is perpendicular to local gravity we will refer to a horizontal trapping model, where $g=0$ in Eq. (1); otherwise, we refer to a vertical trapping model (where the direction of motion will still be $z$ ). By considering a purely one-dimensional model we will be neglecting the possibility for a change in orbital angular momentum in the trap, which could be possible if motion in other directions is also included (for the pure magnetic trap case, see Refs. [11,12]).

An rf-dressed adiabatic potential results from the Hamiltonian of Eq. (1) when either the static or the rf field (or both) vary with position $[1-3,14]$. In this paper we consider a static magnetic field of the form $\mathbf{B}(z)=B(z) \mathbf{e}_{z}$, which can be found, 
for example, on axis in a quadrupole field. The direction of this field is fixed, but the variation of the magnitude of the field with $z$ plays a crucial role in this paper. We take the radio-frequency (rf) magnetic field to have the form $\mathbf{B}_{\mathrm{rf}}(t)=B_{\mathrm{rf}} \cos \left(\omega_{\mathrm{rf}} t\right) \mathbf{e}_{x}$, which gives maximal coupling for a linearly polarized field. Other polarizations are possible without significant change to the details below [2]. The applied rf field induces the atoms to undergo transitions between the different Zeeman states within a single hyperfine spin manifold such that the atoms are confined and forced to oscillate near the location where the frequency of the applied rf field matches the frequency splitting of the Zeeman sublevels [1,2].

In the analysis here the $\mathrm{rf}$ field is treated classically and $B_{\mathrm{rf}}$ is position independent (which is suitable for rf fields generated by macroscopic coils, but not generally suitable for atom chip cases where the rf field is generated "on chip" $[6,15])$. To obtain the adiabatic potentials we first utilize a unitary transformation

$$
\hat{U}_{1}=\exp \left(-i s \frac{\omega_{\mathrm{rf}} t}{\hbar} \hat{F}_{z}\right),
$$

where the quantity $s$ represents the sign of $g_{F}$, i.e.,

$$
s=\frac{g_{F}}{\left|g_{F}\right|} .
$$

Using this spin rotation to change our basis we obtain a transformed Hamiltonian via $\hat{H}_{\text {tot }}^{\prime}=\hat{U}_{1}^{\dagger} \hat{H}_{\text {tot }} \hat{U}_{1}-i \hbar \hat{U}_{1}^{\dagger} \partial_{t} \hat{U}_{1}$. Then, after also making the rotating wave approximation (RWA) we find $[1,2]$

$$
\hat{H}_{\text {tot }}^{\prime}=\frac{\hat{p}_{z}^{2}}{2 M}+\hat{H}_{\mathrm{RWA}}+M g \hat{z},
$$

where

$$
\hat{H}_{\mathrm{RWA}}=s\left[-\delta(\hat{z}) \hat{F}_{z}+\Omega_{0} \hat{F}_{x}\right] .
$$

We note that, in anticipation of Sec. IV, the unitary transformation $\hat{U}_{1}$ commutes with the momentum operator $\hat{p}_{z}$, which leaves the kinetic operator unchanged in Eq. (4). The Rabi frequency $\Omega_{0}$ is used as a measure of the strength of the coupling between the rf field and the atom and is given by $\Omega_{0}=\left|g_{F}\right| \frac{\mu_{B}}{2 \hbar} B_{\mathrm{rf}}$. The detuning, i.e., the frequency difference between the Zeeman split energy levels at $z$ and the rf field frequency of oscillation, is given by

$$
\delta(z)=\omega_{\mathrm{rf}}-\frac{\left|g_{F} \mu_{B} B(z)\right|}{\hbar} .
$$

We will pay attention to the kinetic term in Eq. (4) in Sec. IV A. If we, for now, neglect the kinetic term, and consider the absence of any coupling, i.e., $\Omega_{0} \longrightarrow 0$, we obtain from Eq. (4) the uncoupled or "bare" potentials,

$$
-m_{F} s \hbar \delta(z)+M g z
$$

In the presence of a coupling $\Omega_{0}$, but still neglecting the kinetic term, the Hamiltonian (4) can be diagonalized at any given position $z$. We use a time-independent spin rotation $\hat{U}_{2}$ about the $y$ axis with an angle $\theta$, so that [2]

$$
\hat{U}_{2}=\exp \left(-i \theta(\hat{z}) \hat{F}_{y} / \hbar\right)
$$

where

$$
\theta(z)=\arccos \left(-\frac{\delta(z)}{\sqrt{\delta(z)^{2}+\Omega_{0}^{2}}}\right)+\frac{s-1}{2} \pi
$$

and $s$ is the sign introduced in Eq. (3). Thus we obtain for the Hamiltonian in the adiabatic approximation

$$
\begin{aligned}
\hat{H}_{\text {eff }} & =\hat{U}_{2}^{\dagger} \hat{H}_{\mathrm{RWA}} \hat{U}_{2}+M g \hat{z} \\
& =\sqrt{\delta(\hat{z})^{2}+\Omega_{0}^{2}} \hat{F}_{z}+M g \hat{z},
\end{aligned}
$$

which leads to the adiabatic potentials

$$
V_{m_{F}^{\prime}}(z)=m_{F}^{\prime} \hbar \sqrt{\delta^{2}(z)+\Omega_{0}^{2}}+M g z,
$$

which are trapping potentials for $m_{F}^{\prime}>0$. These potentials form our underlying atom trap, and are illustrated in Fig. 1 for an example with $\delta$ varying linearly in space. To understand the process of decay from these traps we must account for the kinetic term neglected to reach Eq. (11). We will do this in Sec. IV. However, we will first look at the semiclassical Landau-Zener analysis of the situation.

\section{LANDAU-ZENER THEORY}

The original Landau-Zener model $[9,10]$ is a two-level time-dependent model in which the coupling between the two levels is constant and the time-dependent bare potentials change linearly in time. We can apply it to the simplified situation of Fig. 1 by assuming that the spatial potential $\hbar \delta(z)$

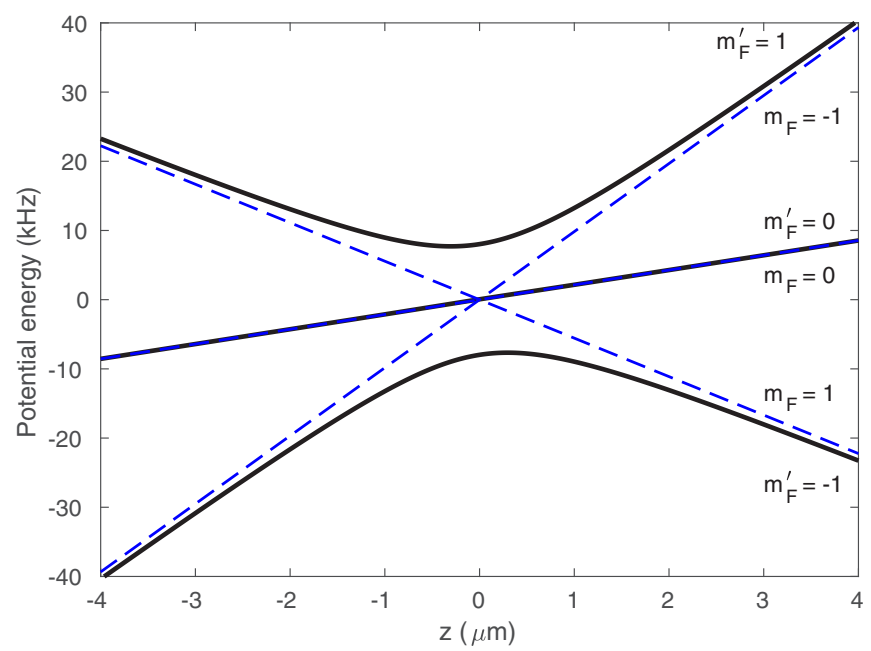

FIG. 1. Bare and adiabatic potentials $V$ as a function of atomic position $z$ for an atom with total angular momentum $F=1$ in an rf-dressed cold-atom trap. The gravitational potential is included and results in the slope of the coincident potentials labeled $m_{F}=0$ and $m_{F}^{\prime}=0$. Blue dashed lines show the potentials given in Eq. (7), with the crossing point necessary for Landau-Zener theory, and labeled $m_{F}=-1,0,1$. The solid black lines show the adiabatic potentials in the dressed state basis given by Eq. (11) and labeled $m_{F}^{\prime}=-1,0,1$. To give a concrete example, we use values taken from Ref. [16], i.e., for a ${ }^{87} \mathrm{Rb}$ atom and a magnetic-field gradient of $B^{\prime}=1.1 \mathrm{~T} / \mathrm{m}$ resulting in a detuning which varies linearly with position. The Rabi frequency is set to $\Omega_{0} / 2 \pi=8 \mathrm{kHz}$ for this graph. 
is linearized about the resonance location at $z=0$ so that

$$
\delta(z) \sim \delta^{\prime}(0) z= \pm \alpha z
$$

where the prime indicates differentiation with respect to $z$ and the " \pm " is to account for a different sign of the gradient of $\delta(z)$ while keeping the magnitude of the gradient, $\alpha$, positive so that $\alpha=\left|\delta^{\prime}(0)\right|$. A constant gradient is, for example, exactly the situation on axis in a quadrupole trap $[13,16]$ where $\alpha=$ $\left|g_{F} \mu_{B} B^{\prime}\right| / \hbar$, with $B^{\prime}=\frac{\partial B}{\partial z}$ being the magnetic-field gradient in the $z$ direction.

The Landau-Zener model assumes that the atom travels at constant speed on a linear potential, at least for the duration of the crucial region around $\delta=0$ (the "crossing point" of the "bare" states in Fig. 1 where there is magnetic resonance). These are very crude approximations, but the model then yields the probability for an atom to make a transition out of the adiabatic state it would be following when it is away from the resonance location. The Landau-Zener model is commonly used to estimate the significance of nonadiabatic losses from rf-dressed cold-atom traps $[14,17]$ by combining the transition probability with the number of crossings per unit time due to the atom oscillating in the adiabatic trap. For the Landau-Zener model, we again neglect the kinetic term in Eq. (4) and taking $F=\frac{1}{2}$, together with a speed $v$ such that $z(t) \sim v t$, we obtain

$$
H_{\mathrm{LZ}}=\frac{\hbar}{2}\left(\begin{array}{cc}
\alpha v t & \Omega_{0} \\
\Omega_{0} & -\alpha v t
\end{array}\right)+M g v t .
$$

Following the Landau-Zener model [1,9,10,18-22], the probability for remaining in the adiabatic state is given by

$$
P_{\mathrm{LZ}}(v)=1-\exp \left(-\frac{\pi \Omega_{0}^{2}}{2 \alpha v}\right)
$$

Gravity does not play a role in this Landau-Zener model as it is assumed that the atom passes through the crossing point at $z=0$ and the factor $M g v t$ just introduces a global phase factor. In the original two-level model there is also an assumption that the atom does not return through the crossing. If it does, there can be interference effects due to the differing phase factors at the crossing $[23,24]$. As we will be working in the adiabatic limit, we will neglect these phase factors, even though the atom will be, in reality, oscillating in the adiabatic potential. The work in Refs. [23,24] shows that the Landau-Zener probability $P_{\mathrm{LZ}}$ still plays a key role.

The standard Landau-Zener model only considers crossings between two energy levels; however, Vitanov and Suominen [25] have extended the model to account for a crossing involving $2 F+1$ energy levels. If we consider first a single pass of the atom through the crossing region, the probability that an atom remains in the initial extremal adiabatic state is given by $P_{\mathrm{LZ}}^{F}(v)=\left[P_{\mathrm{LZ}}(v)\right]^{2 F}$ [25]. We now repeat the argument: every time the atom traverses the crossing, the probability of being lost from the initial adiabatic state is $1-P_{\mathrm{LZ}}^{F}$. Thus, to obtain an estimate of the decay rate, it is necessary only to consider how many times the atom will "pass" the crossing region per unit of time, taking into account that the atom transverses the crossing region twice per period. The decay rate from the Landau-Zener model as a function of atomic speed $v$ is then given by

$$
\Gamma^{\mathrm{LZ}}(v)=\frac{\omega_{z}}{\pi}\left[1-\left[1-\exp \left(-\frac{\pi \Omega_{0}^{2}}{2 \alpha v}\right)\right]^{2 F}\right],
$$

where $\omega_{z}$ is the oscillation frequency in the dressed trap. This can be estimated from the classical motion of a particle in the potential. For a trap which already has a sufficiently strong coupling to be approximately adiabatic, the exponential term is very small and we then obtain the very small decay rate

$$
\Gamma^{\mathrm{LZ}}(v) \approx \frac{2 \omega_{z} F}{\pi} \exp \left(-\frac{\pi \Omega_{0}^{2}}{2 \alpha v}\right) .
$$

Noting that the speed $v$ in Eq. (13) is defined on the bare potentials, we can write Eq. (16) in terms of the approximate total energy $E=\frac{1}{2} M v^{2}$ of the atom in the bare state referenced to zero potential at the crossing, i.e., as

$$
\Gamma^{\mathrm{LZ}}(E) \approx \frac{2 \omega_{z} F}{\pi} \exp \left(-\frac{\pi \Omega_{0}^{2}}{2 \alpha} \sqrt{\frac{M}{2 E}}\right) .
$$

This suggests that for adiabatic trapping a strong Rabi frequency $\Omega_{0}$ is desirable, as is a low gradient $\alpha$ and low energies $E$.

\section{QUANTUM DYNAMICS IN THE 1D TRAP}

To perform a quantum-mechanical analysis of the Hamiltonian (4) and decay from the adiabatic trap we again approximately diagonalize Eq. (4), this time including the kinetic term. We again use the rotation $\hat{U}_{2}$, Eq. (8), and we note that the position dependence of the angle $\theta(z)$, Eq. (9), prevents the unitary transformation $\hat{U}_{2}$ from commuting with the momentum operator. The origin of this is the spatial dependence of the static field amplitude $B(z)$. Thus, to determine the effect of the unitary transformation $\hat{U}_{2}$ on the Hamiltonian (4), we will need to use the relation $\hat{U}_{2}^{\dagger} \hat{p}_{z} \hat{U}_{2}=$ $\hat{p}_{z}-\theta^{\prime}(\hat{z}) \hat{F}_{y}$. As a result, we find that the Hamiltonian for a single atom, already loaded into an rf-dressed cold-atom trap, can be expressed by

$$
\hat{H}=\frac{\hat{p}_{z}^{2}}{2 M}+\hat{V}_{A} \hat{F}_{y}+\hat{V}_{B} \hat{F}_{y}^{2}+\sqrt{\Omega_{0}^{2}+\delta(\hat{z})^{2}} \hat{F}_{z}+M g \hat{z},
$$

where

$$
\begin{aligned}
\hat{V}_{A} & =-\frac{1}{2 M}\left(2 \hat{\theta}^{\prime} \hat{p}_{z}-i \hbar \hat{\theta}^{\prime \prime}\right) \rightarrow \frac{i \hbar}{2 M}\left(2 \theta^{\prime} \frac{\partial}{\partial z}+\theta^{\prime \prime}\right) \\
& =\frac{i \hbar}{2 M}\left[\frac{2 \delta \delta^{\prime 2} \Omega_{0}}{\left(\Omega_{0}^{2}+\delta^{2}\right)^{2}}-\frac{\delta^{\prime \prime} \Omega_{0}}{\Omega_{0}^{2}+\delta^{2}}-\frac{2 \delta^{\prime} \Omega_{0}}{\Omega_{0}^{2}+\delta^{2}} \frac{\partial}{\partial z}\right]
\end{aligned}
$$

and

$$
\hat{V}_{B}=\frac{\left(\hat{\theta}^{\prime}\right)^{2}}{2 M} \rightarrow \frac{1}{2 M} \frac{\delta^{\prime 2} \Omega_{0}^{2}}{\left(\Omega_{0}^{2}+\delta^{2}\right)^{2}},
$$

with a prime indicating differentiation by $z$ and with $\hat{\theta}^{\prime} \equiv \theta^{\prime}(\hat{z})$.

The gauge potential terms given by $\hat{V}_{A} \hat{F}_{y}$ and $\hat{V}_{B} \hat{F}_{y}^{2}$ are often neglected to consider the Hamiltonian in the adiabatic 
approximation. Here we shall use the nonadiabatic Hamiltonian to model the losses from an rf-dressed trap caused by transitions between dressed spin states. Equation (18) applies for general $\delta(z)$, but in the case where $\delta(z)$ is linearized, as in Eq. (12), the first-order derivative is constant and the second-order $\delta^{\prime \prime}$ is zero. In this case we can see from Eqs. (19) and (20) that $\hat{V}_{A}$ is an odd function of $z$ and $\hat{V}_{B}$ is an even function of $z$. As a result, in the treatment below, $\hat{V}_{A}$ will couple states of opposite parity and $\hat{V}_{B}$ will couple states of the same parity. Expressing the Hamiltonian as

$$
\begin{aligned}
\hat{H}= & \frac{\hat{p}_{z}^{2}}{2 M}+\sqrt{\Omega_{0}^{2}+\delta(\hat{z})^{2}} \hat{F}_{z}+M g \hat{z}+\frac{\hat{V}_{A}}{2 i}\left(\hat{F}_{+}-\hat{F}_{-}\right) \\
& +\frac{\hat{V}_{B}}{2}\left(\hat{F}^{2}-\hat{F}_{z}^{2}\right)-\frac{\hat{V}_{B}}{4}\left(\hat{F}_{+}^{2}+\hat{F}_{-}^{2}\right),
\end{aligned}
$$

where $\hat{F}_{ \pm}=\hat{F}_{x} \pm i \hat{F}_{y}$ and $\hat{F}^{2}=\hat{F}_{x}^{2}+\hat{F}_{y}^{2}+\hat{F}_{z}^{2}$, it can be seen that $\hat{V}_{A}$ gives the coupling between states with $\left|\Delta m_{F}^{\prime}\right|=1$ and $\hat{V}_{B}$ gives both an energy shift and the coupling between states with $\left|\Delta m_{F}^{\prime}\right|=2$. For an $F=1$ system, as displayed in Fig. 1 with the trapping potential defined as the case $m_{F}^{\prime}=1$, the $\hat{V}_{A}$ coupling then induces transitions to the $m_{F}^{\prime}=0$ spin state and the $\hat{V}_{B}$ coupling induces transitions to the $m_{F}^{\prime}=-1$ spin state. Once in the $m_{F}^{\prime}=0$ or $m_{F}^{\prime}=-1$ states, the atoms are highly likely to travel out of the trapping region and be permanently lost from the trap.

\section{A. Quantum-mechanical nonadiabatic decay rates}

In this section formulas for the rate of nonadiabatic spin "flips" out of a rf-dressed cold-atom trap are obtained using Fermi's golden rule. This is justified in the situation where the nonadiabatic effects act as a perturbation on the adiabatic states.

In the following development we consider two cases: first the horizontal trapping model (Sec. IV B), where the orientation of the motion, perpendicular to gravity, ensures that gravity plays no role in the dynamics. Secondly, we consider in Sec. IV C a vertical trapping model where gravity acts to pull atoms out of the region of rf resonance and to modify their oscillation frequency. Interactions between the atoms are not considered, making this analysis unsuitable for Bose-Einstein condensates but reasonable for dilute atomic clouds comprised of thermal atoms. In the following analytic development, for simplicity, we also neglect the $\hat{V}_{B}$ coupling term as numerical investigations have shown it to have a small effect for the parameters of interest. However, some of the numerical results presented do include a contribution to the $m_{F}^{\prime}=0,1$ potentials from the $\hat{V}_{B}$ term: this contribution, a nonadiabatic potential, is described in the Appendix.

We consider a trapped atom with $F=1$ and calculate decay rates for the rate of transitions from the $m_{F}^{\prime}=1$ dressed spin state to the $m_{F}^{\prime}=0$ dressed spin state. With these assumptions we use perturbation theory to derive equations which model the rate of transitions between dressed spin states in an rf-dressed cold-atom trap. Our analysis can be extended to other spin systems, but note that for $F>1$ transitions from the extremal trapping potential $\left(m_{F}^{\prime}=F\right)$ would not be to a continuum, as in the case $F=1$, but to $m_{F}^{\prime}=F-1$ which will have discrete states.
Thus the unperturbed Hamiltonian for the system is taken to be

$$
\hat{H}_{0}=\frac{\hat{p}_{z}^{2}}{2 M}+\sqrt{\Omega_{0}^{2}+\delta(\hat{z})^{2}} \hat{F}_{z}+M g \hat{z},
$$

with the perturbing term given by $\Delta \hat{H}=\hat{V}_{A} \hat{F}_{y}$. The initial trapping potential is then given by $V_{i}(z)=\hbar \sqrt{\Omega_{0}^{2}+\delta(z)^{2}}+M g z$ (where $g=0$ in the horizontal trapping model). For the untrapped $m_{F}^{\prime}=0$ dressed spin state the final adiabatic potential will be set to $V_{f}(z)=M g z$ (where again $g=0$ in the horizontal trapping model). The origin of the $z$ axis is the resonance location, with $\delta(z=0)=0$.

For the next sections we define the following notation where we use the product state $\left|F=1, m_{F}^{\prime}=1\right\rangle \cdot\left|\Phi_{n}\right\rangle$ for the $n$th eigenfunction of a trapped atom which is composed of spin states $\left|F=1, m_{F}^{\prime}=1\right\rangle$ and spatial states $\left|\Phi_{n}\right\rangle$ such that the spatial wave function $\Phi_{n}(z)$ is given by $\Phi_{n}(z)=$ $\left\langle z \mid \Phi_{n}\right\rangle$. Similarly, for the untrapped spin state we will use the product state $\left|F=1, m_{F}^{\prime}=0\right\rangle \cdot\left|\Psi_{k}\right\rangle$ where the label $k$ will be associated with the outgoing momentum of the escaping atom and the wave function $\Psi_{k}(z)$ will be given by $\Psi_{k}(z)=\left\langle z \mid \Psi_{k}\right\rangle$.

\section{B. Horizontal trapping model}

We first consider a situation where there is in effect no gravitational potential, such as when the trapping is in the horizontal direction. This is the case in a ring trap $[7,8]$ where rf adiabatic potentials provide horizontal confinement and optical light shifts provide vertical confinement. The situation could also arise in a microgravity environment, or when gravity is compensated with other fields.

A harmonic approximation of the potential $\hbar \sqrt{\delta(z)^{2}+\Omega_{0}^{2}}$ for the trapped $m_{F}^{\prime}=1$ state can be obtained by Taylor expansion to give

$$
V_{i}(z)=\hbar \Omega_{0}+\frac{1}{2} M \omega_{z}^{2} z^{2}
$$

with a trapping frequency

$$
\omega_{z}=\alpha \sqrt{\frac{\hbar}{M \Omega_{0}}} .
$$

Here we have used Eq. (12), $\delta(z)= \pm \alpha z$, with positive $\alpha$, and we expect the expansion to be valid in the region $|z| \ll w$, where $w$ characterizes the range of the rf interaction and is given by $w=\Omega_{0} / \alpha$. The $n$th wave function for an atom in the initial trapped $m_{F}^{\prime}=1$ spin state is given by the usual harmonic-oscillator wave function

$$
\Phi_{n}(z)=\frac{H_{n}\left(z / a_{z}\right)}{\sqrt{n ! 2^{n} a_{z} \sqrt{\pi}}} e^{-z^{2} /\left(2 a_{z}^{2}\right)},
$$

with the associated energy $E_{n}=\left(n+\frac{1}{2}\right) \hbar \omega_{z}+\hbar \Omega_{0} . H_{n}$ is the Hermite polynomial of degree $n$ and the variable $a_{z}=$ $\sqrt{\hbar /\left(M \omega_{z}\right)}$ is the standard length scale associated with the harmonic-oscillator frequency $\omega_{z}$.

For our untrapped $m_{F}^{\prime}=0$ state, in the absence of gravity the potential for the atoms is zero, i.e.,

$$
V_{f}(z)=0 \text {. }
$$

However, to allow a calculation of the density of states, we consider the system to be confined to a region 
$-L / 2<z<L / 2$, later taking $L \rightarrow \infty$. Starting from the harmonic state $\left|\Phi_{n}\right\rangle$, with a parity $(-1)^{n}$ set by the index $n$ of the harmonic-oscillator eigenfunction, the wave function $\Psi_{k}(z)$ of the final state is then given by

$$
\Psi_{k}(z)=\frac{1}{\sqrt{2 L}}\left[e^{i k z}-(-1)^{n} e^{-i k z}\right],
$$

where $k$ stands for $k(n)$ and depends on the initial state. The factor $(-1)^{n}$ ensures that the final state and the initial state coupled by the operator $\hat{V}_{A}$ have opposite parity as discussed in Sec. IV. When we apply Fermi's golden rule the energy $E_{k(n)}=$ $\hbar^{2} k^{2}(n) /(2 M)$ must match the harmonic-oscillator energy of the initial state, i.e., $E_{n}=\left(n+\frac{1}{2}\right) \hbar \omega_{z}+\hbar \Omega_{0}$. Thus we have

$$
k(n) a_{z}=\sqrt{1+2 n+2 \Omega_{0} / \omega_{z}},
$$

where $n$ corresponds to the index of the initial state $\left|\Phi_{n}\right\rangle$. In the following we introduce $q(n)$, a scaled momentum, which stands for

$$
q(n)=k(n) a_{z}=\sqrt{1+2 n+2 \eta^{2}},
$$

with the dimensionless variable $\eta=w / a_{z}$ being the LandauZener crossing length scale $w$ scaled to the harmonic-oscillator length scale $a_{z}$. Its expression as a function of $\Omega_{0}$ and $\alpha$ reads

$$
\eta=\left(\frac{M}{\hbar}\right)^{1 / 4} \frac{\Omega_{0}^{3 / 4}}{\alpha^{1 / 2}}
$$

To apply Fermi's golden rule we need the density of states $D(E)=\frac{\partial N}{\partial E}$ which is given by

$$
D(E)=\frac{1}{2} \frac{L}{\pi \hbar} \sqrt{\frac{M}{2 E}}=\frac{M L}{2 \pi \hbar^{2} k(n)},
$$

where a factor of one-half arises because we only select states with appropriate parity. Then, putting this all together, the Fermi's golden rule decay rate for an atom with initial state $\left|\Phi_{n}\right\rangle$ with energy $E_{n}$ in the horizontal trapping model is

$$
\begin{aligned}
\Gamma_{n}= & \left|\left\langle m_{F}^{\prime}=1\left|\hat{F}_{y}\right| m_{F}^{\prime}=0\right\rangle\right|^{2} \lim _{L \rightarrow \infty} \frac{M L}{2 \pi \hbar^{2} k} \frac{2 \pi}{\hbar}\left|\int_{-\frac{L}{2}}^{\frac{L}{2}} \Phi_{n}^{*}(z) \hat{V}_{A} \Psi_{k}(z) d z\right|^{2} \\
= & \omega_{z} \frac{w^{2} a_{z}}{2^{n+2} n ! k \sqrt{\pi}} \mid\left\{\int_{-\infty}^{\infty} \frac{z H_{n}\left(z / a_{z}\right) e^{-\frac{z^{2}}{2 a_{z}^{2}}}}{\left(z^{2}+w^{2}\right)^{2}}\left[e^{i k z}+(-1)^{n+1} e^{-i k z}\right] d z\right. \\
& \left.-i k \int_{-\infty}^{\infty} \frac{H_{n}\left(z / a_{z}\right) e^{-\frac{z^{2}}{2 a_{z}^{2}}}}{z^{2}+w^{2}}\left[e^{i k z}+(-1)^{n} e^{-i k z}\right] d z\right\}\left.\right|^{2},
\end{aligned}
$$

where we have used $\left|\left\langle m_{F}^{\prime}=1\left|\hat{F}_{y}\right| m_{F}^{\prime}=0\right\rangle\right|^{2}=\hbar^{2} / 2$. In Eq. (31) $k$ stands for $k(n)$. Equation (31) is written using the dimensionless parameters $q$ and $\eta$ as

$$
\Gamma_{n}=\omega_{z} \frac{\eta^{2}}{2^{n+2} n ! q \sqrt{\pi}}\left|\int_{-\infty}^{\infty} d u H_{n}(u) e^{-u^{2} / 2}\left[\frac{u\left(e^{i q u}+(-1)^{n+1} e^{-i q u}\right)}{\left(u^{2}+\eta^{2}\right)^{2}}-i \frac{q\left(e^{i q u}+(-1)^{n} e^{-i q u}\right)}{u^{2}+\eta^{2}}\right]\right|^{2},
$$

where $q$ stands for $q(n)$, as given in Eq. (28). Numerical results obtained from Eq. (32) are presented on Fig. 2 (and also on Fig. 4 which will be discussed in the next Sec. IV C).

An analytical solution for the ground-state case with $n=0$ can be found where $\left[H_{0}(u)=1\right]$. To accomplish this we use the integrals 3.954.1 and 3.954.2 from Gradshteyn and Ryzhik [26] to find

$$
\Gamma_{0}=\omega_{z} \frac{\pi^{\frac{3}{2}}}{16 q_{0}} e^{\eta^{2}}\left\{e^{-\eta q_{0}}\left(q_{0}+\eta\right) \operatorname{erfc}\left[\frac{-1}{\sqrt{2}}\left(q_{0}-\eta\right)\right]+e^{\eta q_{0}}\left(q_{0}-\eta\right) \operatorname{erfc}\left[\frac{1}{\sqrt{2}}\left(q_{0}+\eta\right)\right]\right\}^{2},
$$

which is expressed in terms of "erfc," the complementary error function [27], the dimensionless variable $\eta$, and the scaled dimensionless momentum $q_{0}=k_{(n=0)} a_{z}=\sqrt{2 \eta^{2}+1}$. We see that $q_{0}$ depends on $\eta$ and that $q_{0}>\eta$.

For higher-energy trapped atoms with $n \geqslant 1$ the integrals contained within Eq. (32) can be approximated in the region $\eta \gtrsim 5$ by calculating the residue of a pole found within them. This leads to an analytic expression for the decay rates provided by Fermi's golden rule for any $n$ state [28]:

$$
\begin{aligned}
\Gamma_{n} \approx & \omega_{z} \frac{\pi^{\frac{3}{2}}}{2^{n+2} n ! q} \exp \left(\eta^{2}-2 \eta q\right) \\
& \times\left|2 n H_{n-1}(i \eta)-i(q+\eta) H_{n}(i \eta)\right|^{2},
\end{aligned}
$$

where, again, $q$ stands for $q(n)=k(n) a_{z}$, Eq. (28), which does depend on $\eta$. Results from this expression are shown for a specific example in Fig. 2 where good agreement is seen with the numerical evaluation of Eq. (32) (solid line in that figure).

It is useful to find the $\Omega_{0} \rightarrow \infty$ and $B^{\prime} \rightarrow 0$ (or equivalently $\alpha \rightarrow 0$ ) limits of Eq. (34), as it is in these regimes that coldatom traps favorably operate as the losses due to nonadiabatic effects are low there. Since $\Gamma_{n} / \omega_{z}$ depends only on $\eta$ and $n$ in Eq. (34), and since $\eta \propto \Omega_{0}^{3 / 4} / \alpha^{1 / 2}$, see Eq. (29), both limits are found from $\eta \rightarrow \infty$. The limiting behavior, valid for states $n$ such that $n \ll \eta^{2}$, is then

$$
\Gamma_{n} / \omega_{z} \underset{\eta \rightarrow \infty}{\sim} \frac{2^{n}}{n !} \eta^{2 n+1} e^{-\sqrt{2}\left(n+\frac{1}{2}\right)} e^{-(2 \sqrt{2}-1) \eta^{2}} .
$$




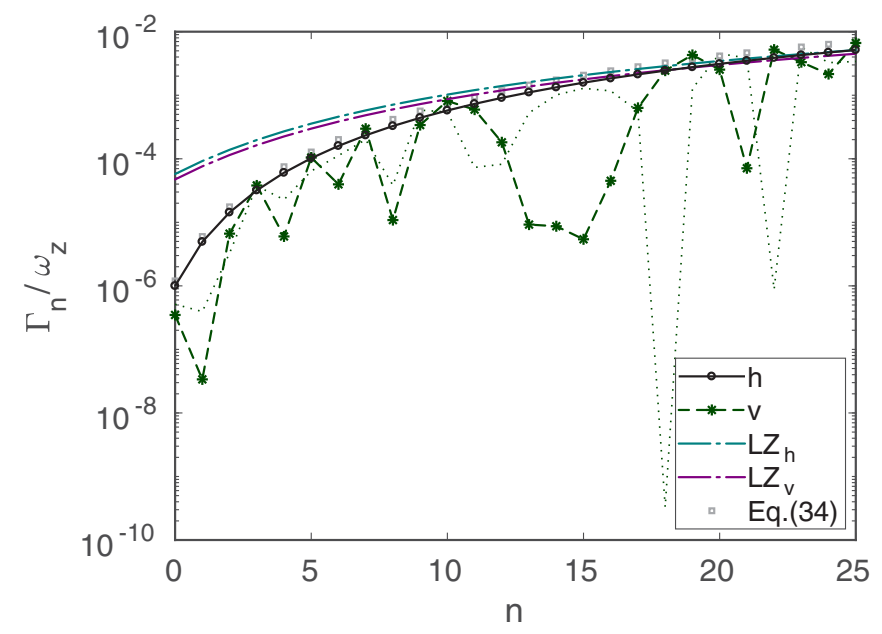

FIG. 2. Scaled decay rate $\Gamma_{n} / \omega_{z}$ is shown as a function of the harmonic-oscillator label $n$ for different models. The solid line with circles shows the result for the horizontal trapping model $(h)$ as given by Eq. (32). The dashed line with asterisks indicates the scaled vertical trapping decay rates $(v)$ as given by Eq. (47). These vertical trapping decay rates are not a smooth function of $n$. We can also include nonadiabatic potentials based on including part of $\hat{V}_{B}$ and resulting in modified parameters given in the Appendix. For the vertical trapping model, the effect is a shift of the location of the "dips" (dotted line). However, in the case of the horizontal trapping model, the inclusion of these corrections produces no visible change to the solid $(h)$ line and is not shown. The chained lines $\left(\mathrm{LZ}_{h}\right.$ and $\left.\mathrm{LZ}_{v}\right)$ indicate the result of a Landau-Zener calculation given by Eq. (51) (with $\epsilon$ set to zero in the " $h$ " case). For the parameters used (i.e., from Fig. 1) the trap frequency was $\omega_{z} / 2 \pi=0.93 \mathrm{kHz}$ for the horizontal trapping model [Eq. (23)] and corresponding Landau-Zener model. For the case of the vertical trapping model and its corresponding Landau-Zener curve, the trap frequency is found from Eq. (39) to be $\omega_{z} / 2 \pi=0.87 \mathrm{kHz}$. The points marked with small squares are given by the analytic approximation Eq. (34). The calculations are done for the $F=1$ hyperfine ground state of ${ }^{87} \mathrm{Rb}$ with the parameters given in Fig. 1. This corresponds to $\eta=\Omega_{0} /\left(\alpha a_{z}\right) \sim 2.9$ for the horizontal trapping model and, for the vertical trapping model, $\eta \sim 2.8$ [Eq. (49)] and $\epsilon=M g /(\hbar \alpha)=$ 0.28 .

We will see in Sec. V that this behavior agrees qualitatively with our semiclassical interpretation (Sec. III). The trap frequency (23) is increased for high magnetic-field gradient or low Rabi frequency which raises the energy of the $n$th oscillator level and increases crossing speed. Additionally, tighter trapping potentials lead to the orientation of the local effective magnetic-field direction changing more rapidly over a given distance. Both of these factors result in a greater probability for an atom to become misaligned from the local effective magnetic-field vector, leading to greater nonadiabatic losses as $\Omega_{0} \rightarrow 0$ or $B^{\prime} \rightarrow \infty$. Equation (35) is useful for specifying the main dependence of nonadiabatic decay rates on $\Omega_{0}$ and $B^{\prime}$, additionally indicating that the process is more sensitive to Rabi frequency than magnetic-field gradient since $\eta^{2} \propto \Omega_{0}^{3 / 2} / B^{\prime}$.

\section{Vertical trapping model}

In the case where the $z$ axis is oriented vertically we can no longer neglect the effect of gravity on the location of the equilibrium position of the atoms in the adiabatic potential. This time the harmonic expansion yields an initial trapping potential

$$
V_{i}(z)=V_{0}+\frac{1}{2} M \omega_{z}^{2}\left(z-z_{0}\right)^{2},
$$

where, because of gravity, the center of the harmonic oscillator is shifted from the origin (where there is resonance) to a point

$$
z_{0}=-\frac{\epsilon \Omega_{0}}{\alpha \sqrt{1-\epsilon^{2}}}
$$

below the origin. The parameter $\epsilon=M g / \hbar \alpha$ is introduced as the ratio of the gravitational force to the force applied by the magnetic-field gradient. The approximate harmonic potential now has a modified energy offset [compared to Eq. (22)]

$$
V_{0}=\hbar \Omega_{0} \sqrt{1-\epsilon^{2}}
$$

and a modified trap frequency [16]

$$
\omega_{z}=\alpha \sqrt{\frac{\hbar}{M \Omega_{0}}}\left(1-\epsilon^{2}\right)^{\frac{3}{4}} .
$$

We see that if $\epsilon=0$ we recover the horizontal trapping model result Eq. (23), and also that there is no trap unless $\epsilon<1$. This condition is equivalent to gravity compensation by the magnetic force in the underlying static magnetic trap, namely $\hbar \alpha>M g$.

The wave function for an atom in the initial trapped $m_{F}^{\prime}=1$ state is now a displaced harmonic-oscillator wave function,

$$
\Phi_{n}(z)=\frac{H_{n}\left[\left(z-z_{0}\right) / a_{z}\right]}{\sqrt{n ! 2^{n} a_{z} \sqrt{\pi}}} e^{-\left(z-z_{0}\right)^{2} /\left(2 a_{z}^{2}\right)},
$$

where $a_{z}=\sqrt{\hbar / M \omega_{z}}$ should be used with the appropriate trap frequency $\omega_{z}$, Eq. (39), and $n$ is a positive integer which selects the energy of the trapped atom from the allowed discrete harmonic-oscillator energy levels given by the relevant $E_{n}=$ $\left(n+\frac{1}{2}\right) \hbar \omega_{z}+V_{0}$.

The potential for an untrapped atom $\left(m_{F}^{\prime}=0\right)$ is now simply

$$
V_{f}(z)= \begin{cases}\infty, & z \leqslant-L, \\ M g z, & z>-L .\end{cases}
$$

To assist with the calculation we have introduced a distance $L$ to a (single) hard wall of the potential, see Fig. 3, similar to the distance $L$ in Sec. IV B. Later we will also let $L \rightarrow \infty$. The corresponding stationary Schrödinger equation in the final state $m_{F}^{\prime}=0$ is given by

$$
E_{\kappa} \Psi_{\kappa}(z)=-\frac{\hbar^{2}}{2 M} \frac{d^{2} \Psi_{\kappa}(z)}{d z^{2}}+M g z \Psi_{\kappa}(z),
$$

where $\kappa$ is an index labeling the final state. Equation (42) can be written in terms of a second-order partial differential equation for a spatially shifted Airy function, $\frac{d^{2}}{d \zeta^{2}} \operatorname{Ai}(\zeta)=\zeta \operatorname{Ai}(\zeta)$, where the argument $\zeta$ is given by $\zeta=\tilde{z}-\tilde{z}_{\kappa}$ and we use the scaled distance $\tilde{z}=z / \ell$. The characteristic length of the Airy function is linked to the atom mass and gravity through

$$
\ell=\left(\frac{\hbar^{2}}{2 M^{2} g}\right)^{1 / 3}
$$




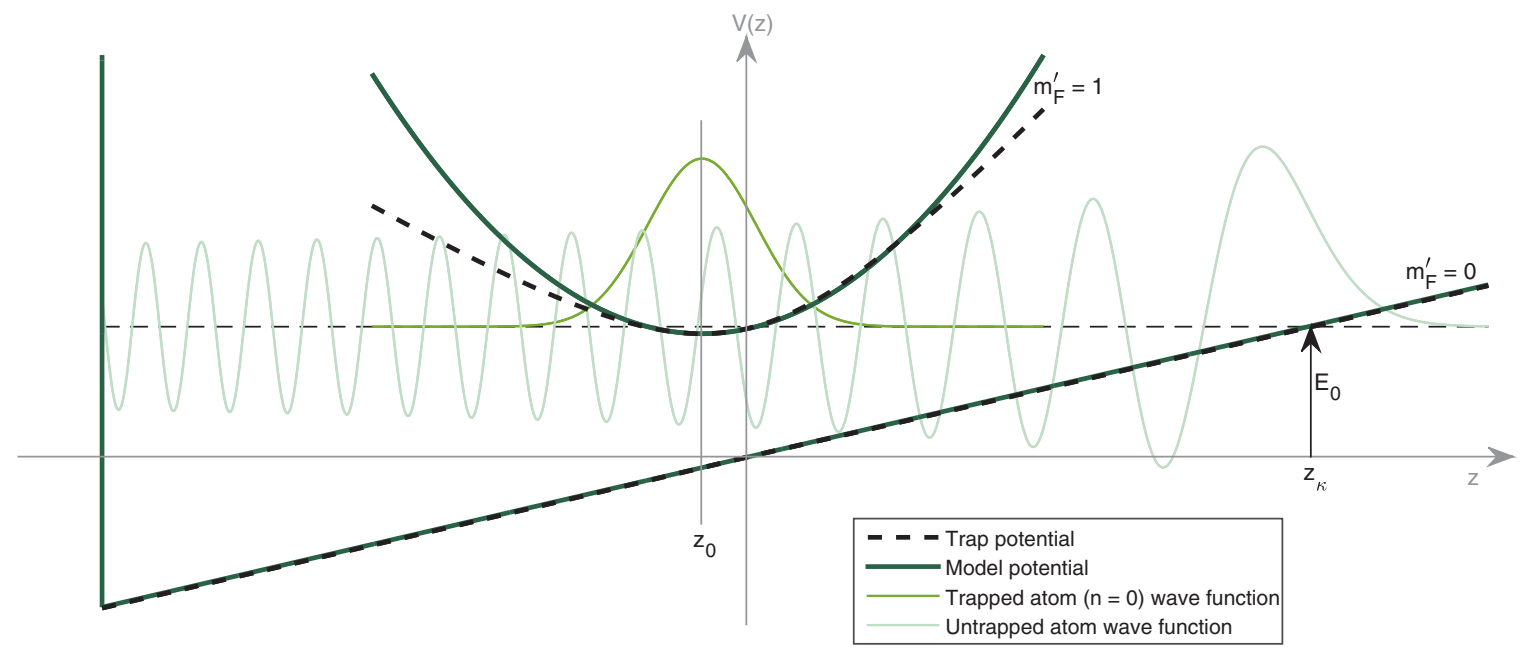

FIG. 3. Schematic diagram showing the key wave functions and potentials $V_{i, f}(z)$ in the vertical trapping model. The wave functions are scaled to equal maximum height for comparison. The ground-state wave function of the harmonic oscillator is shown centered at $z_{0}$, Eq. (37), in its potential $V_{i}(z)$, Eq. (36). The energy of the ground state is $E_{0}$, as given by $V_{0}+\hbar \omega_{z} / 2$, Eqs. (38),(39). An energy resonant Airy function is shown, which is an eigenstate of the linear potential $V_{f}(z)$, Eq. (41). The potential "wall" on the left is located at $z \rightarrow-\infty$ in the calculations. The eigenstate is associated with a turning point at $z_{\kappa}$. The parameters for this figure are as in Fig. 1.

and the turning point for a classical particle is at $z_{\kappa}=$ $E_{\kappa} / M g=\tilde{z}_{\kappa} \ell$. Fermi's golden rule will require energy matching of the $m_{F}^{\prime}=0$ and $m_{F}^{\prime}=1$ states as seen for $n=0$ in Fig. 3. In terms of $z$, the solution of the stationary Schrödinger equation for the untrapped state is then $[27,29]$

$$
\Psi_{\kappa}(z)=\mathcal{C} \operatorname{Ai}\left[\left(z-z_{\kappa}\right) / \ell\right],
$$

where $\mathcal{C}$ is a normalization constant and $\mathrm{Ai}$ is the Airy function of the first kind.

The normalization constant $\mathcal{C}$ for the untrapped state wave function can be determined from the condition that $|\mathcal{C}|^{2} \int_{-L}^{\infty}\left|\operatorname{Ai}\left[\left(z-z_{\kappa}\right) / \ell\right]\right|^{2} d z=1$. If the Airy function $\operatorname{Ai}(\zeta)$ is approximated in the $\zeta \rightarrow-\infty$ limit by [27] $\operatorname{Ai}(\zeta) \approx$ $\frac{1}{\sqrt{\pi}(-\zeta)^{\frac{1}{4}}} \sin \left[\frac{2}{3}(-\zeta)^{\frac{3}{2}}+\frac{\pi}{4}\right]$, this leads to an approximate normalization constant for the untrapped state wave function given by $|\mathcal{C}|^{2} \approx \pi / \sqrt{\ell L}$. In the continuum limit, and to apply Fermi's golden rule, we need the density of states. To determine this we note that the potential wall at $z=-L$ creates a boundary condition for the wave function as $\Psi_{\kappa}(-L)=0$. The asymptotic form of the Airy function can be used to express this condition as a quantization condition since the argument of the sine function should be a multiple of $\pi$. Specifying the multiple by the integer $n_{\kappa}$, we have the condition $n_{\kappa} \pi=\frac{2}{3}\left[\left(z_{\kappa}+L\right) / \ell\right]^{\frac{3}{2}}+\frac{\pi}{4}$. Differentiation of this quantization condition, together with $E_{\kappa}=M g z_{\kappa}$, leads to an equation for the density of states,

$$
\begin{aligned}
D\left(E_{\kappa}\right) & =\frac{\partial n_{\kappa}}{\partial E_{\kappa}}=\frac{1}{\pi(M g \ell)^{3 / 2}} \sqrt{E_{\kappa}+M g L} \\
& \simeq \frac{1}{\pi M g \ell} \sqrt{\frac{L}{\ell} .}
\end{aligned}
$$

It is noteworthy that the $L$ dependence cancels in the product $|\mathcal{C}|^{2} \cdot D\left(E_{\kappa}\right)=1 /\left(M g \ell^{2}\right)$ such that there are no issues when taking the $L \rightarrow \infty$ limit.
The interaction matrix element associated with the nonadiabatic coupling is given by

$$
\frac{\sqrt{2} \hbar}{2 i} \int_{-\infty}^{\infty} \Phi_{n}^{*}(z) \hat{V}_{A} \Psi_{\kappa}(z) d z
$$

where, as in the horizontal trapping model, the factor $2 i$ arises from the component of $\hat{F}_{-}$in $\hat{F}_{y}$ [see Eq. (18)] and the factor $\sqrt{2} \hbar$ comes from the matrix element of $\hat{F}_{-}$between $m_{F}^{\prime}=1$ and $m_{F}^{\prime}=0$. In evaluating this integral the wave functions will be given by Eqs. (40) and (44).

All the components necessary for use of Fermi's golden rule are now known, and putting this together we find the decay rate for the $n$th oscillator state in the vertical trapping model

$$
\begin{aligned}
\Gamma_{n}= & \omega_{z} \frac{2 \sqrt{\pi} \eta^{2}}{n ! 2^{n} \beta} \\
& \times \mid \int_{-\infty}^{\infty} \frac{\left(u+u_{0}\right) H_{n}(u) e^{-\frac{u^{2}}{2}}}{\left[\left(u+u_{0}\right)^{2}+\eta^{2}\right]^{2}} \operatorname{Ai}\left[\beta\left(u+u_{0}-u_{\kappa}\right)\right] d u \\
& -\left.\beta \int_{-\infty}^{\infty} \frac{H_{n}(u) e^{-\frac{u^{2}}{2}}}{\left(u+u_{0}\right)^{2}+\eta^{2}} \operatorname{Ai}^{\prime}\left[\beta\left(u+u_{0}-u_{\kappa}\right)\right] d u\right|^{2},
\end{aligned}
$$

where we integrate over $u=\left(z-z_{0}\right) / a_{z}$ and we have defined $u_{0}=z_{0} / a_{z}, u_{\kappa}=z_{\kappa} / a_{z}=E_{\kappa} /\left(M g a_{z}\right), E_{\kappa}=E_{n}, \beta=$ $a_{z} / \ell$, and $\eta=\Omega_{0} /\left(\alpha a_{z}\right)$. (Note that $\eta, \omega_{z}$, and $a_{z}$ differ from the expressions of Sec. IV B.) The function $\mathrm{Ai}^{\prime}$ is the usual derivative of the Airy function with respect to its argument. This result for $\Gamma_{n} / \omega_{z}$ can be expressed solely in terms of $n, \eta$, and $\epsilon$, since the integrals in (47) depend only on $n, \eta, \beta, u_{0}$, 

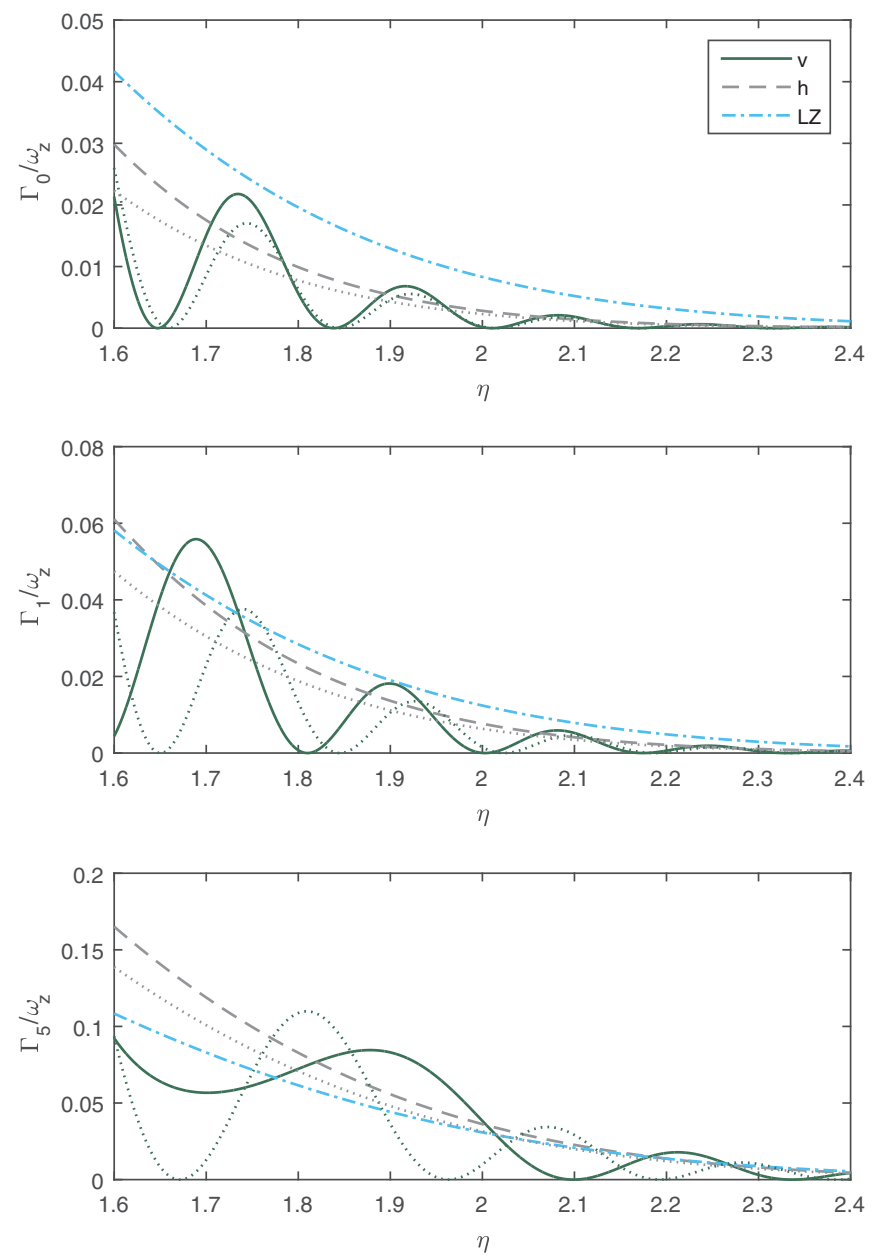

FIG. 4. Scaled decay rates $\Gamma_{n} / \omega_{z}$ as a function of the parameter $\eta$ for the ground state $\left(\Gamma_{0} / \omega_{z}\right.$, top), the first excited state $\left(\Gamma_{1} / \omega_{z}\right.$, middle), and the fifth excited state $\left(\Gamma_{5} / \omega_{z}\right.$, bottom) of the initial harmonic trap. The prediction of the vertical trapping model, Eq. (47), solid line, is shown with the predictions of the horizontal trapping model, Eq. (32), dashed line, and the Landau-Zener model, Eq. (51), chained line. The dotted lines indicate the effect of nonadiabatic potentials which produce corrections to $\omega_{z}$ from Eq. (A9) and to $\Gamma_{n}$ as described in Appendix 2 and in Fig. 2. The calculations are done for the $F=1$ hyperfine ground state of ${ }^{87} \mathrm{Rb}$ with $\epsilon \sim 0.20$ in the vertical trapping model case.

and $u_{\kappa}$, and with the above definitions it can be shown that

$$
\begin{aligned}
\beta^{3} & =\left(a_{z} / \ell\right)^{3}=2 \eta \epsilon\left(1-\epsilon^{2}\right)^{-3 / 2}, \\
u_{0} & =z_{0} / a_{z}=-\eta \epsilon\left(1-\epsilon^{2}\right)^{-1 / 2}, \\
u_{\kappa} & =\frac{V_{0}+(n+1 / 2) \hbar \omega_{z}}{M g a_{z}} \\
& =\frac{\eta}{\epsilon}\left(1-\epsilon^{2}\right)^{1 / 2}\left[1+\frac{1-\epsilon^{2}}{\eta^{2}}\left(n+\frac{1}{2}\right)\right] .
\end{aligned}
$$

Gravitational effects are very weak if $\epsilon$ is small $(\epsilon \ll 1)$ and we see in Sec. $\mathrm{V}$ that the adiabatic limit is reached if $\eta$ is large $(\eta \gg 1)$.

Figures 2 and 4 show numerical results obtained from Eq. (47) for the scaled decay rate $\Gamma_{n} / \omega_{z}$ as a function of the initial quantum number $n$ in the harmonic trap and of the parameter $\eta$ given in the general case by

$$
\eta=\left(\frac{M \Omega_{0}^{3}}{\hbar \alpha^{2}}\right)^{1 / 4}\left(1-\epsilon^{2}\right)^{3 / 8}
$$

We note that we have approximately $\eta \propto \Omega_{0}^{3 / 4} B^{\prime-1 / 2}$ when $\epsilon$ stays small (as in the horizontal trapping case). The parameter $\eta$ thus remains more sensitive to the Rabi frequency, proportional to the amplitude of the dressing field, than to the magnetic gradient.

In contrast to the predictions of the horizontal trapping model, the vertical trapping model displays in Fig. 4 a clear oscillatory behavior in the decay rate variation with $\eta$, or equivalently when varying Rabi frequency or magnetic-field gradient. Additionally, in contrast to the semiclassical interpretation of nonadiabatic losses, there is not a monotonic increase of the decay rate with atomic energy or vibrational level $n$, with some high $n$ states being sheltered from nonadiabatic losses.

To help understand the origin of the oscillatory behavior of the decay rates, Fig. 5 shows the shape of the trapped and untrapped state wave functions for two different Rabi frequencies. The vertical lines marked $z_{0}$ are nearly coincident in Fig. 5 indicating that the center of the harmonic oscillator

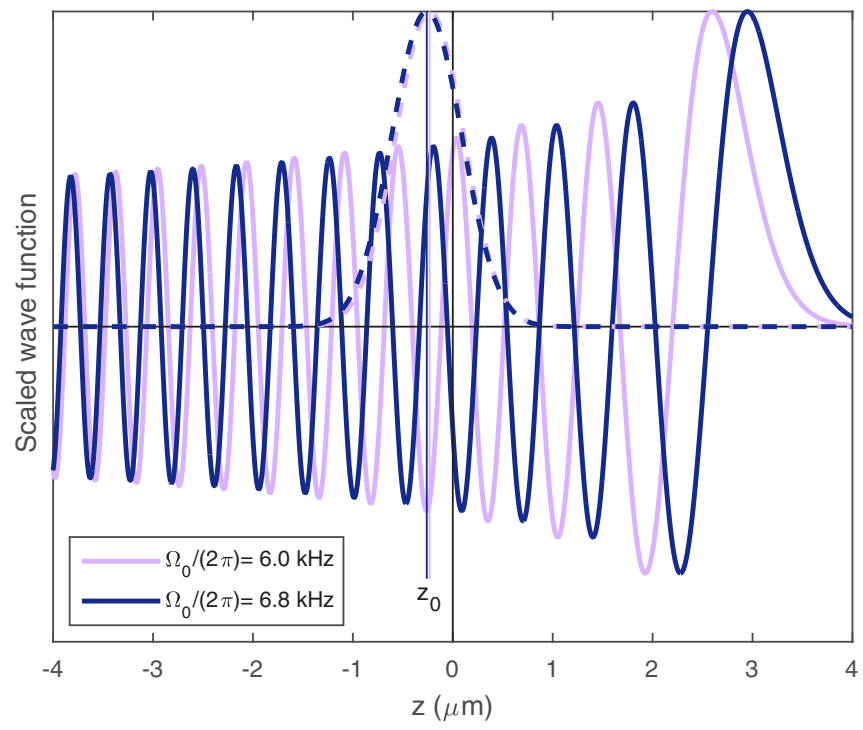

FIG. 5. Examination of the sensitivity of the wave functions in the vertical trapping model. The trapped harmonic-oscillator groundstate wave function $\Phi_{n}(z)$, associated with $m_{F}^{\prime}=1$, is displayed with dashed lines for two different Rabi frequencies: $\Omega_{0} /(2 \pi)=6.0 \mathrm{kHz}$ and $6.8 \mathrm{kHz}$. Other parameters are fixed and are the same as in Fig. 1. Also shown are the two Airy functions associated with $m_{F}^{\prime}=0$ eigenstates matching the energies of the corresponding harmonicoscillator ground states. We see that there is a substantial shift in the peaks of the Airy functions between the two Rabi frequencies shown, whilst the change in the location of the oscillator ground state, $z_{0}$, is very small. This shows how the overlap integral Eq. (46) can be sensitive to parameters. The wave functions have been scaled so that they reach a value of unity at the maximum height. 
hardly shifts when the Rabi frequency is changed. However, the energy of the untrapped state is set to match that of the trapped atom as needed to satisfy Fermi's golden rule. This means that changing the harmonic-oscillator energy-level structure (for example, by changing the Rabi frequency) affects the Airy wave function. Thus, when the Rabi frequency is changed, we see that although the displacement in the minimum $z_{0}$ is weakly affected, the oscillations of the relevant Airy function are significantly displaced. It is this progression of the Airy function peaks which leads to the oscillatory behavior in the decay rates obtained as a function of $\eta$ (and $\epsilon$ ) and seen in Fig. 4. (This oscillatory dependence on $\eta$ can also be found equivalently as a function of the unscaled parameters $\Omega_{0}$ or $B^{\prime}$.) In other words, for the case of horizontal trapping, the phase of a plane wave can always be set to match the location of the atom, but in the case of vertical trapping, the oscillatory phase of the Airy function is restricted by the energy of the initial state. For vertical trapping, the behavior of $\Gamma_{n}$ as a function of $n$ is not smooth as there is a dramatic change in the harmonic-oscillator wave function $\left(\Phi_{n}\right)$ with the quantum number $n$, which affects the interaction matrix element and leads to the results seen in Fig. 2.

\section{COMPARISON OF QUANTUM DYNAMICS WITH THE LANDAU-ZENER MODEL}

In this section we compare the quantum decay rates obtained in Sec. IV to the Landau-Zener model introduced in Sec. III. Returning to Eq. (15), we note that the concept of atomic speed relies on the idea of a classical trajectory. We stress here that the classical trajectory in the bare (uncoupled) potential should be used to compute the transition rate [20]. Although the trajectory is described classically, for a more direct comparison with our quantum mechanical decay rates it is beneficial to describe the Landau-Zener decay rate in terms of the atomic energy level denoted by the quantum number $n$. By considering energy conservation of an atom at the resonance location, the expression $\frac{1}{2} M v^{2}=E_{n}=V_{0}+$ $\hbar \omega_{z}\left(n+\frac{1}{2}\right)$ is obtained, which leads to

$$
v=\sqrt{\frac{2 V_{0}}{M}+(2 n+1) \omega_{z}^{2} a_{z}^{2}}
$$

for the atomic speed through the resonance location. Therefore, the Landau-Zener decay rate (16) for an $F=1$ atom in the $n$th harmonic-oscillator energy level is

$$
\Gamma_{n}^{\mathrm{LZ}}=\frac{\omega_{z}}{\pi}\left\{1-\left[1-\exp \left(-\frac{\pi \eta^{2}}{2 \sqrt{2}\left(1-\epsilon^{2}\right) \sqrt{1+\frac{1}{\eta^{2}}\left(n+\frac{1}{2}\right)\left(1-\epsilon^{2}\right)}}\right)\right]^{2}\right\}
$$

In the limit $\epsilon \rightarrow 0$ we obtain the result for horizontal trapping, that is

$$
\Gamma_{n}^{\mathrm{LZ}}=\frac{\omega_{z}}{\pi}\left\{1-\left[1-\exp \left(-\frac{\pi \eta^{2}}{2 \sqrt{2} \sqrt{1+\frac{1}{\eta^{2}}\left(n+\frac{1}{2}\right)}}\right)\right]^{2}\right\} .
$$

To simplify the expression further we note that for the lowest harmonic levels, where the harmonic approximation for the adiabatic potential is valid ( $n \ll \eta^{2}$ and $\eta \gg 1$ ), we obtain

$$
\Gamma_{n}^{\mathrm{LZ}} \simeq \frac{2 \omega_{z}}{\pi} e^{(2 n+1) \pi /(8 \sqrt{2})} \exp \left(-\frac{\pi \eta^{2}}{2 \sqrt{2}}\right) .
$$

Comparison of Eq. (35) with the Landau-Zener model limiting behavior given in Eq. (53) shows some structural similarity, but also clear differences between our model and the LandauZener model in the low decay regime. The similarity is the exponential dependence on $\eta^{2}$ with a slight difference in the multiplying factors, i.e., a factor of $\pi /(2 \sqrt{2}) \simeq 1.1$ in the Landau-Zener case, and a factor of approximately 1.8 in the case of Eq. (35). For large $\eta$, the desired limit for trap operation, this exponential dependence is the most dominating aspect and leads to an overestimation of the decay rate by the Landau-Zener model. Another difference between the results, which is more relevant at lower $\eta$, is that the polynomial prefactor, with its power-law dependence $\eta^{2 n+1}$, is absent in the Landau-Zener model.
The overestimation of the Landau-Zener model is seen in Fig. 2. In particular, it is most clearly seen for the vibrational ground state $(n=0)$, and lower $n$ values, which are often dominantly populated at the low temperatures necessary for ultracold atom traps. The Landau-Zener result improves in comparison with the higher $n$ values of the horizontal model in Fig. 2; however, when comparing it to the vertical model there are the irregular oscillations in the decay rate as discussed in Sec. IV C. These kinds of oscillations cannot be obtained from the simple application of the Landau-Zener model to a single crossing. However, although the Landau-Zener model generally overestimates the decay rate, there are a few points, at higher $n$, where the oscillatory vertical trapping model decay rate slightly exceeds the Landau-Zener result.

Figure 6 shows how the Fermi golden rule decay rates imply less stringent requirements on trap Rabi frequency and magnetic-field gradient in comparison to the Landau-Zener model prediction for a given ground-state lifetime. Here we use the direct experimental parameters $\Omega_{0}$ and $B^{\prime}$ to clearly indicate the practical consequences of the results for ${ }^{87} \mathrm{Rb}$. We see that the Landau-Zener model provides useful guidance: given the logarithmic scales of the figure, the powerlaw dependence of Landau-Zener result on the parameters is approximately correct, with a consistent margin. When adiabaticity is reduced below the Landau-Zener boundary in Fig. 6 the oscillatory structures of the vertical trapping model appear. The details of these are sensitive to phase shifts from nonadiabatic corrections to the potentials, as seen from the dotted lines. 


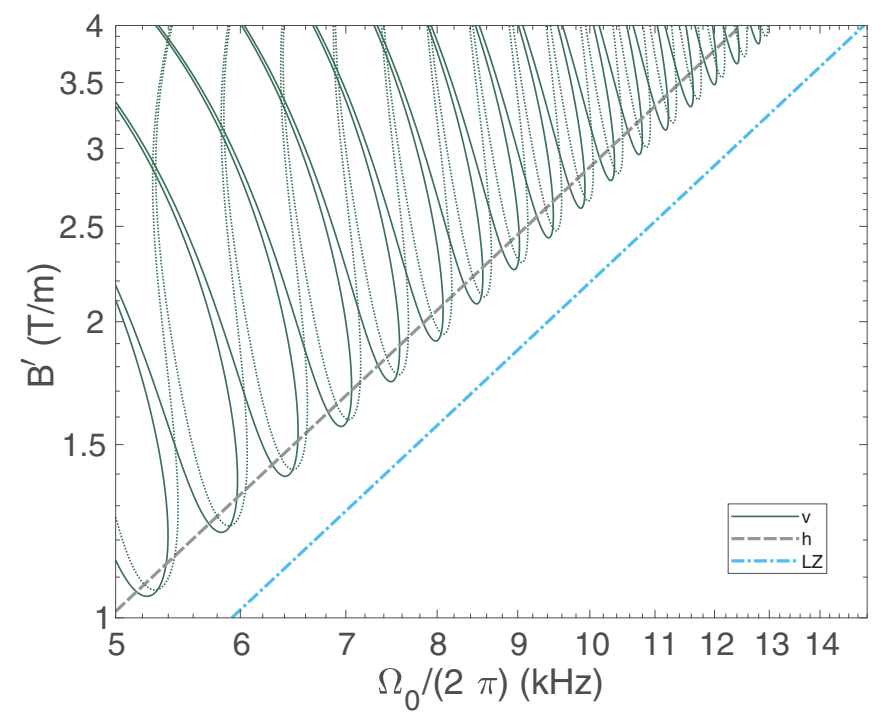

FIG. 6. Ground-state decay rate contours for different models as a function of Rabi frequency and field gradient. The contours show where the scaled decay rate $\Gamma_{0} / \omega_{z}=10^{-3}$, i.e., where decay takes place after about 160 oscillations in the trap. The decay rates generally increase towards the top left of the figure. The solid contour line $(v)$ shows the result from the vertical trapping model, as given by Eq. (47). The dotted contour line shows the effect of including the nonadiabatic potentials from $\hat{V}_{B}$ in the vertical trapping model case (see Appendix 2). The dashed contour line corresponds to the ground-state decay rate from the horizontal trapping model $(h)$, as given by Eq. (32). It is a suitable approximation to the boundary displayed by the vertical trapping model. The effect of including the nonadiabatic potentials is not shown for the horizontal trapping model as there is no visible difference from the dashed contour line. The chained contour line corresponds to the Landau-Zener decay rate, Eq. (51), with a trap frequency calculated using Eq. (39). The results presented in this figure are for ${ }^{87} \mathrm{Rb}$ and $F=1$.

\section{CONCLUSION}

Since their experimental inception in 2004 [5], rf-dressed adiabatic potentials for cold atoms have been successfully applied to a wide variety of situations [1]. Generally speaking, for the trap to work effectively, one is content to ensure that a sufficiently strong rf field is employed so that as few atoms as possible are lost from the trap. However, if an overstrong rf field is used the trapping frequency of the trap itself is reduced, which is undesirable if low dimensionality is required [30]. Furthermore, an overstrong rf field can result in infringement of the RWA [31]. And there may be other situations, for example, involving time-averaged adiabatic potentials or multifrequency adiabatic potentials [1,2], where the Rabi frequency is required to be constrained. Then the design question arises as to how small the coupling can be made before the trap is no longer effective.

In this paper we have tried to address this complex situation with a number of significant approximations, but also with the aim of obtaining some analytic results. We have had to treat a one-dimensional quantum problem as the three-dimensional problem is not separable due to the changing direction of gravity and the typical change in relative orientation of the magnetic and rf fields around the full 3D trapping surface [2]. The approximation seems reasonable for those cases where the atom cloud is sufficiently compact. We have assumed that the rf polarization is linear, and in a specific direction. However, in this case the results can be generalized quite easily to the case of other polarizations. We have also assumed that the atomic Rabi frequency is uniform for the spatial $z$ variation that we consider. As discussed, this is a reasonable approximation for some situations (macroscopic coils for rf radiation), but needs more careful consideration in other cases such as when atom chips are being used. We have also assumed the linear Zeeman effect (though for a treatment of adiabatic potentials in the nonlinear regime, see Ref. [32]). The final expressions for decay rates have assumed a local harmonic approximation for the adiabatic potential. This assumption places a constraint on the excitation in the trapping degree of freedom (i.e., $n \ll \eta^{2}$ ). Of course, this constraint could be relaxed in a fully numerical approach to the problem where the harmonic-oscillator basis states are not used in the evaluation of the matrix elements.

Fermi's golden rule (i.e., time-dependent perturbation theory) has been a key tool to determine the decay rates. This relies on a weak coupling between the initial and final states of the model and is expected to be very appropriate in the adiabatic limit. However, in assuming that the coupling is weak we have neglected the possibility of non-Markovian dynamics during the decay process. This is reasonable as such dynamics would only be expected to appear when the atom loss is very rapid and it would be difficult to observe. We have also focused on the case $F=1$ in this paper. Aspects of the derivation can be easily generalized to higher $F$; however, it may not be possible to use Fermi's golden rule any more because the $\hat{V}_{A}$ couplings are no longer to a continuum when the uppermost adiabatic state $\left(m_{F}^{\prime}=F, F>1\right)$ is considered for nonadiabatic loss. However, the good news is that it is likely that because the transitions are now bound-bound rather than bound-continuum the loss of atoms from the uppermost adiabatic state is expected to be strongly inhibited; that is, you would be "unlucky" to find a coincidence of the vibrational eigenenergies of $m_{F}^{\prime}=F$ and $m_{F}^{\prime}=F-1$ because the harmonic frequencies are not commensurate with each other and are also shifted by an incommensurate Rabi frequency. We remark, however, that the role of the transverse ( $x$ and $y$ ) directions, which we do not take into account here, could compensate for this energy mismatch and restore the losses. An additional approximation in the work presented here is the neglect of that part of the $\hat{V}_{B}$ coupling that causes a downward change in $m_{F}^{\prime}$ by two. In the case of $F=1$ this would add the complication of an additional density of final states on an inverted $\left(m_{F}^{\prime}=-1\right)$ potential as well as possible second-order $\hat{V}_{A}$ processes that could coherently interfere with it. Numerical work has suggested that these effects can be neglected for the parameters we have considered here, but the effects could become significant in other situations and it would be good to quantify this in the future. However, the part of the $\hat{V}_{B}$ coupling that causes nonadiabatic potentials to be added to the $m_{F}^{\prime}$ states has been included. These are based on the analysis in the Appendix and included as dotted lines for $\Gamma_{n}$ in Figs. 2, 4, and 6. Finally, in this recapitulation of the approximations, we have not included consideration here of some very practical matters such as the losses due to collisions 
with background gas atoms and molecules or the effects of heating due to noise in the currents that may produce either the static magnetic field or the rf magnetic field.

Despite these approximations, the results for horizontal trapping, Eq. (34), and vertical trapping, Eq. (47), should be able to offer some safe guidance with an appropriate estimate for the excitation $n$, or with a distribution of $n$ such as can be found with a thermal state. To obtain the results we used both Landau-Zener theory and Fermi's golden rule with an $F=1$ spin system in a dressed atom trap with an underlying linear magnetic-field gradient.

The Landau-Zener model in general overestimates nonadiabatic transitions, particularly for the ground state. However, for this (good result) it is essential to use the correct speed in the Landau-Zener expression (16) as discussed in Sec. III. For practical purposes it is satisfactory to use the simpler Landau-Zener expressions, where possible, and be able to err on the side of safety.

The basic results for $\Gamma / \omega_{z}$ can be expressed in terms of a single dimensionless parameter $\eta$ for the horizontal trapping model, or in terms of two parameters $\eta$ and $\epsilon$ for the vertical trapping model. In the fuller and richer vertical model, oscillatory behavior is seen in the Fermi golden rule treatment for the decay rate as a function of both magnetic-field gradient and Rabi frequency. As a result, and counter to intuition with the Landau-Zener model, higher-energy states do not necessarily lead to higher decay rates at all places in the parameter space.

In conclusion, we believe that the analytic results and procedures presented here will be useful in the design and testing of atom traps based on adiabatic potentials. In particular, the analytic approximate expression given at Eq. (34) gives a good estimate of the expected loss rate and is easily calculated even for large values of $\eta$, where the exact formula Eq. (32) is harder to compute numerically. More generally, Fig. 6 gives an indication, in terms of laboratory-based parameters rather than dimensionless variables, of the parameter region to avoid based on the analysis and approximations used here when applied to ${ }^{87} \mathrm{Rb}$ and $F=1$. The generalization to higher $F$ and relaxation of some of the approximations listed above would be useful in future work.

\section{ACKNOWLEDGMENTS}

The authors would like to thank Germán Sinuco-Leon for helpful discussions and careful reading of the manuscript. This work was supported by the Leverhulme Trust and by EPSRC Grants No. EP/I010394/1 and No. EP/M013294/1. We acknowledge financial support from ANR project SuperRing (No. ANR-15-CE30-0012-01). LPL is a member of Institut Francilien de Recherche sur les Atomes Froids (IFRAF).

\section{APPENDIX: EFFECT OF NONADIABATIC POTENTIALS}

In this appendix we present again the key steps of the development of the decay rates for horizontal and vertical trapping, but this time we keep the effect of a small nonadiabatic potential which is presented as the dotted lines for $\Gamma_{n}$ in Figs. 2, 4, and 6. We start by considering the Hamiltonian (18), which is already in the adiabatic basis. As explained in Sec. IV this can be slightly rearranged to give Eq. (21) with two $\hat{V}_{B}$ terms: one which couples states which have a difference in $m_{F}^{\prime}$ of two units (proportional to $\hat{F}_{+}^{2}+\hat{F}_{-}^{2}$ ) and one term which does not change $m_{F}^{\prime}$ and which is proportional to $\hat{F}^{2}-\hat{F}_{z}^{2}$. It is the effect of the latter term which we focus on in this appendix. As it will not change $m_{F}^{\prime}$ the term will cause a spatially dependent energy shift in the adiabatic potentials, but only in the presence of nonadiabatic loss. This makes the observation of this nonadiabatic potential very challenging as its effects are only seen significantly when atoms are lost quickly from an adiabatic trap. Nevertheless, similar kinds of effects are discussed in the context of three-level Raman systems in Ref. [33].

Thus, neglecting the $\hat{V}_{B}$ term which changes $m_{F}^{\prime}$ in Eq. (21), but keeping the $\hat{V}_{B}$ energy shift term proportional to $\hat{F}^{2}-\hat{F}_{z}^{2}$ we start with the Hamiltonian

$$
\begin{aligned}
\hat{H}= & \frac{\hat{p}_{z}^{2}}{2 M}+\hat{V}_{A} \hat{F}_{y}+\frac{\hat{V}_{B}}{2}\left(\hat{F}^{2}-\hat{F}_{z}^{2}\right)+{\sqrt{\Omega_{0}^{2}}}^{2} \\
& +\delta(\hat{z})^{2} \hat{F}_{z}+M g \hat{z},
\end{aligned}
$$

such that the contribution to the adiabatic potentials from the $\hat{V}_{B}$ term is considered. As in Sec. IV we consider that we have a kinetic term $\hat{p}_{z}^{2} /(2 M)$, a perturbative term $\hat{V}_{A} \hat{F}_{y}$, which is used for the coupling in Fermi's golden rule, and a remaining part which forms the adiabatic potentials and which now includes a $\hat{V}_{B}$ term proportional to $\hat{F}^{2}-\hat{F}_{z}^{2}$. Because of the presence of the $\hat{V}_{B}$ term in Eq. (A1) the adiabatic potentials (11) are now replaced by

$$
V_{m_{F}^{\prime}}(z)=\hbar m_{F}^{\prime} \sqrt{\Omega_{0}^{2}+\delta^{2}(z)}+\Xi \frac{\Omega_{0}^{2} \delta^{\prime}(z)^{2}}{\left(\Omega_{0}^{2}+\delta^{2}(z)\right)^{2}}+M g z,
$$

where in the linear regime we have a uniform gradient $\delta^{\prime}$, Eq. (12). As in Sec. II the sign term $s=g_{F} /\left|g_{F}\right|$ is absorbed into $m_{F}^{\prime}$ and the dressed spin states which correspond to trapping potentials are defined to have positive $m_{F}^{\prime}$ values. We have introduced a parameter

$$
\Xi=\frac{\hbar^{2}\left[F(F+1)-m_{F}^{\prime 2}\right]}{4 M},
$$

which characterizes the scale of the new contribution from $\hat{V}_{B} \hat{F}_{y}^{2}$ to the adiabatic potentials for a particular atomic species. The parameter $\Xi$ is strictly a function of $m_{F}^{\prime}$ (and $F$ ), but as we consider here the particular case $F=1$ with initial $m_{F}^{\prime}=1$ and final $m_{F}^{\prime}=0$ there will be two values of $\Xi$ which play a role in this appendix: $\Xi_{0}=\hbar^{2} /(2 M)$ and $\Xi_{1}=\Xi_{0} / 2$. The remaining part of the $\Xi$ term in Eq. (A2) comes from $\hat{V}_{B}$, as given in Eq. (20). Thus the term that multiplies $\Xi$ in Eq. (A2) adds a small positive contribution to all adiabatic potentials, regardless of dressed spin state, in the vicinity of the resonance location. The effect of this contribution can be reasonably ignored in the limit $\eta \rightarrow \infty$, the limit in which cold-atom traps favorably operate. However, Figs. 2, 4, and 6 show that there is a noticeable effect on the decay rates when we enter deeply into the nonadiabatic region. 


\section{Nonadiabatic potentials and the horizontal trapping model}

In the horizontal trapping model we do not need to consider the effect of gravitational potential energy and we can express the trapping potential as a harmonic oscillator centered around the resonant detuning location, such that the trapping potential is given by

$$
V_{i}(z)=\hbar \Omega_{0}+\frac{\Xi_{1}}{w^{2}}+\frac{1}{2} M \omega_{z}^{2} z^{2} .
$$

The trap frequency is altered by the $\hat{V}_{B}$ contribution such that it is now given by

$$
\omega_{z}=\sqrt{\frac{\hbar \alpha^{2}}{M \Omega_{0}}-\frac{4 \Xi_{1}}{M w^{4}}}
$$

or equivalently in terms of $\eta=w / a_{z}$ and for $F=1$,

$$
\omega_{z}=\frac{\Omega_{0}}{\eta^{2}}\left(1+\frac{1}{\eta^{4}}\right)^{-1 / 2} .
$$

Here $a_{z}=\sqrt{\hbar /\left(M \omega_{z}\right)}$ is defined using the modified trap frequency given in Eq. (A5). The contribution of the $\Xi$ term could turn the curvature of the harmonic-oscillator potential negative setting a lower limit for acceptable Rabi frequencies: $\Omega_{0}>\left[4 \alpha^{2} \Xi_{1} / \hbar\right]^{\frac{1}{3}}$. This gives $\Omega_{0} / 2 \pi>1.8 \mathrm{kHz}$ for $B^{\prime}=1 \mathrm{~T} / \mathrm{m}$ and $\Omega_{0} / 2 \pi>5.2 \mathrm{kHz}$ for $B^{\prime}=5 \mathrm{~T} / \mathrm{m}$ for the state $|1,1\rangle$ of ${ }^{87} \mathrm{Rb}$.

Following the same approach as in Sec. IV B the wave function $\Phi_{n}(z)$ for a trapped atom is given by Eq. (24) with the modified $a_{z}$, and $n$ selects the energy of the trapped atom from the allowed energy levels which are given by the modified expression $E_{n}=\left(n+\frac{1}{2}\right) \hbar \omega_{z}+\hbar \Omega_{0}+\Xi_{1} / w^{2}$.

For the untrapped $\left|F, m_{F}^{\prime}=0\right\rangle$ state, i.e., in the absence of gravity, the potential for the atoms is an infinite square well which is given by

$$
V_{f}(z)= \begin{cases}\infty, & z \leqslant-\frac{L}{2}, \\ \frac{\Xi_{0}}{w^{2}}, & -\frac{L}{2} \ll z \ll \frac{L}{2}, \\ \infty, & z \geqslant \frac{L}{2} .\end{cases}
$$

The wave function of the final state is given by $\Psi_{k}(z)=$ $\frac{1}{\sqrt{2 L}}\left[e^{i k z}-(-1)^{n} e^{-i k z}\right]$ [as in Eq. (26)] with discrete energy levels $E_{k(n)}=\frac{\hbar^{2} k^{2}(n)}{2 M}+\frac{\Xi_{0}}{w^{2}}$. The wave number $k(n)$ is altered by the inclusion of the $\hat{V}_{B}$ contribution such that

$$
\begin{aligned}
q(n) & =k(n) a_{z}=\sqrt{1+2 n+2 \Omega_{0} / \omega_{z}-1 /\left(2 \eta^{2}\right)} \\
& =\sqrt{1+2 n+2 \eta^{2}+5 /\left(2 \eta^{2}\right)}
\end{aligned}
$$

[which can be compared to Eq. (28)]. Then the Fermi's golden rule decay rate for an atom with energy $E_{n}$ in the horizontal trapping model is given by Eq. (32) with the replacements described above for $\omega_{z}, a_{z}, \eta$, and $q(n)$, which are all affected by the inclusion of the $\Xi$ term in the model.

It then follows that the analytical solution for the groundstate decay rate $\Gamma_{0}$ is given by Eq. (33) but with modified variables $q_{0}=q(0), \eta$, and $a_{z}$ and the approximate decay rate for higher-energy trapped atoms with $n>0$ is the similarly modified Eq. (34).

\section{Nonadiabatic potentials and the vertical trapping model}

In the vertical trapping model case we must keep the gravitational term in the adiabatic potential (A2) and again make a harmonic approximation about the minimum point. When we include the $\Xi$ term there is the complication of the additional dependence on $\delta(z)$, even when $\delta(z)$ is linearized. Thus we again take $V_{i}(z)=V_{0}+\frac{1}{2} M \omega_{z}{ }^{2}\left(z-z_{0}\right)^{2}$, where $z_{0}$ is at the center of the displaced atom cloud which can be determined from $\left.\frac{d}{d z} V_{m_{F}^{\prime}=1}(z)\right|_{z_{0}}=0$. As a result of the new terms in the adiabatic potential the value of $z_{0}$ differs from Eq. (37). The harmonic potential now has energy offset

$$
V_{0}=\hbar \sqrt{\Omega_{0}^{2}+\delta_{0}^{2}}+\Xi_{1} \frac{\Omega_{0}^{4}}{w^{2}\left(\Omega_{0}^{2}+\delta_{0}^{2}\right)^{2}}-\hbar \epsilon \delta_{0},
$$

where $\delta_{0}$ is the detuning at the center of the displaced atom cloud at $z_{0}$. It also has a trap frequency

$$
\omega_{z}=\sqrt{\frac{\Omega_{0}^{4}}{M w^{2}}\left[\frac{\hbar}{\left(\Omega_{0}^{2}+\delta_{0}^{2}\right)^{\frac{3}{2}}}+\frac{4 \Xi_{1} \Omega_{0}^{2}}{w^{2}} \frac{\left(5 \delta_{0}^{2}-\Omega_{0}^{2}\right)}{\left(\Omega_{0}^{2}+\delta_{0}^{2}\right)^{4}}\right]}
$$

which cannot be written in terms of $\eta$ and $\epsilon$ alone because the value of $\delta_{0}$ must be found numerically as described above. All these changes to the potential of the initial state modify the initial wave function $\Phi_{n}(z)$ used in the overlap integral Eq. (46).

The potential for an untrapped atom is now approximated by a modified Eq. (41):

$$
V_{f}(z)= \begin{cases}\infty, & z \leqslant-L, \\ \frac{\Xi_{0}}{w^{2}}+M g z, & z>-L .\end{cases}
$$

The stationary Schrödinger equation for the untrapped state can be written as

$$
E_{\kappa}^{\prime} \Psi_{\kappa}(z)=-\frac{\hbar^{2}}{2 M} \frac{d^{2} \Psi_{\kappa}(z)}{d z^{2}}+\operatorname{Mg} z \Psi_{\kappa}(z),
$$

where the $E_{\kappa}^{\prime}$ is the modified $E_{\kappa}$ of Eq. (42) and is given by $E_{\kappa}^{\prime}=E_{\kappa}-\Xi_{0} / w^{2}$.

The untrapped state wave function is once again given by $\Psi_{\kappa}(z)=\mathcal{C} \operatorname{Ai}(\zeta)=\mathcal{C A i}\left[\left(z-z_{\kappa}\right) / \ell\right]$, but where now $z_{\kappa}=$ $E_{\kappa}^{\prime} /(M g)$. The density of states and normalization coefficient are calculated as before such that $|\mathcal{C}|^{2} \cdot D\left(E_{\kappa}^{\prime}\right)=1 /\left(M g \ell^{2}\right)$. Thus with the modified $\Psi_{\kappa}(z)$ and the modified wave function $\Phi_{n}(z)$ described above we can obtain a modified matrix element from Eq. (46): $(\sqrt{2} \hbar /(2 i)) \int_{-\infty}^{\infty} \Phi_{n}^{*}(z) \hat{V}_{A} \Psi_{\kappa}(z) d z$. Then when we apply Fermi's golden rule we find that the decay rate $\Gamma_{n}$ is given by a modified Eq. (47), but with the further modified parameters $\beta=a_{z} / \ell, \omega_{z}, a_{z}$, and $z_{0}$. These parameters were all affected by the inclusion of the $\hat{V}_{B}$ contribution to the adiabatic potentials in Eq. (A2).
[1] B. M. Garraway and H. Perrin, J. Phys. B: At., Mol., Opt. Phys. 49, 172001 (2016).
[2] H. Perrin and B. M. Garraway, in Advances in Atomic, Molecular, and Optical Physics, edited by E. Arimondo, C. C. 
Lin, and S. F. Yelin (Academic Press, New York, 2017), Vol. 66, Chap. 4, p. 181

[3] O. Zobay and B. M. Garraway, Phys. Rev. Lett. 86, 1195 (2001).

[4] Y. Colombe, B. Mercier, H. Perrin, and V. Lorent, in Proceedings of the Euroschool on Quantum Gases in Low Dimensions, Les Houches 2003, edited by L. Pricoupenko, H. Perrin, and M. Olshanii [J. Phys. IV France 116, 247 (2004)].

[5] Y. Colombe, E. Knyazchyan, O. Morizot, B. Mercier, V. Lorent, and H. Perrin, Europhys. Lett. 67, 593 (2004).

[6] T. Schumm, S. Hofferberth, L. M. Andersson, S. Wildermuth, S. Groth, I. Bar-Joseph, J. Schmiedmayer, and P. Krüger, Nat. Phys. 1, 57 (2005).

[7] O. Morizot, Y. Colombe, V. Lorent, H. Perrin, and B. M. Garraway, Phys. Rev. A 74, 023617 (2006).

[8] W. H. Heathcote, E. Nugent, B. T. Sheard, and C. J. Foot, New J. Phys. 10, 043012 (2008).

[9] L. D. Landau, Phys. Z. Sowjetunion 2, 46 (1932).

[10] C. Zener, Proc. R. Soc. A 137, 696 (1932).

[11] C. V. Sukumar and D. M. Brink, Phys. Rev. A 56, 2451 (1997).

[12] D. M. Brink and C. V. Sukumar, Phys. Rev. A 74, 035401 (2006).

[13] J. Fortágh and C. Zimmermann, Rev. Mod. Phys. 79, 235 (2007).

[14] O. Zobay and B. M. Garraway, Phys. Rev. A 69, 023605 (2004).

[15] J. Reichel and V. Vuletic, Atom Chips (Wiley, New York, 2011).

[16] K. Merloti, R. Dubessy, L. Longchambon, A. Perrin, P.-E. Pottie, V. Lorent, and H. Perrin, New J. Phys. 15, 033007 (2013).

[17] O. Morizot, C. L. Garrido Alzar, P.-E. Pottie, V. Lorent, and H. Perrin, J. Phys. B: At., Mol., Opt. Phys. 40, 4013 (2007).

[18] M. S. Child, Molecular Collision Theory (Academic Press, New York, 1974).
[19] K.-A. Suominen, B. M. Garraway, and S. Stenholm, Opt. Commun. 82, 260 (1991).

[20] B. M. Garraway and S. Stenholm, Opt. Commun. 83, 349 (1991).

[21] B. M. Garraway and K.-A. Suominen, Rep. Prog. Phys. 58, 365 (1995).

[22] N. V. Vitanov and B. M. Garraway, Phys. Rev. A 53, 4288 (1996).

[23] B. M. Garraway and S. Stenholm, Phys. Rev. A 45, 364 (1992).

[24] B. M. Garraway and N. V. Vitanov, Phys. Rev. A 55, 4418 (1997).

[25] N. V. Vitanov and K.-A. Suominen, Phys. Rev. A 56, R4377 (1997).

[26] I. S. Gradshteyn and I. M. Ryzhik, Table of Integrals, Series and Products, 6th ed. (Academic Press, New York, 2000).

[27] M. Abramowitz and I. A. Stegun, Handbook of Mathematical Functions (US GPO, Washington, DC, 1972).

[28] K. A. Burrows, Ph.D. thesis, University of Sussex, Brighton, 2016.

[29] F. Gerbier, P. Bouyer, and A. Aspect, Phys. Rev. Lett. 86, 4729 (2001).

[30] Quantum Gases in Low Dimensions, edited by L. Pricoupenko, H. Perrin, and M. Olshanii, special issue of J. Phys. IV France 116, 1-280 (2004).

[31] S. Hofferberth, B. Fischer, T. Schumm, J. Schmiedmayer, and I. Lesanovsky, Phys. Rev. A 76, 013401 (2007).

[32] G. A. Sinuco-León and B. M. Garraway, New J. Phys. 14, 123008 (2012).

[33] M. Łącki, M. A. Baranov, H. Pichler, and P. Zoller, Phys. Rev. Lett. 117, 233001 (2016). 